\title{
Control estructural de agrietamientos poligonales en la Pedriza del Manzanares (Madrid) ${ }^{1}$
}

\author{
Manuel GARCÍA-RODRÍGUEZ \\ Departamento de Ciencias Analíticas \\ Universidad Nacional de Educación a Distancia (UNED) \\ manu.garo@ccia.uned.es \\ Javier AROZTEGUI \\ Departamento de Psicología Evolutiva y de la Educación \\ Universidad Complutense de Madrid (UCM) \\ jarozteg@ucm.es \\ Haday LÓPEZ PORTILLO \\ Departamento de Tecnología Industrial \\ Universidad Alfonso Décimo el Sabio (UAX) \\ hadaylp@gmail.com
}

Recibido: 31 de agosto del 2015

Enviado a evaluar: 2 de septiembre del 2015

Aceptado: 25 de noviembre del 2015

\section{RESUMEN}

Los agrietamientos poligonales representan una forma común de modelado granítico cuyo origen y evolución continúa en fase de estudio, no existiendo una sistematización de estas estructuras diversas. Algunos autores explican su origen por procesos geodinámicos internos, relacionándolo con movimientos de planos de fractura en estados tardíos de consolidación magmática. Otros autores atribuyen su formación y desarrollo a factores externos relacionados con el régimen climático. La gran variedad de agrietamientos poligonales requiere la utilización de un número mayor de variables para definir los distintos orígenes, y las posibles interrelaciones entre los factores externos e internos, así como para explicar la evolución de dichas estructuras y avanzar en la clasificación de los patrones concretos. Este trabajo pretende contribuir a sistematizar los mecanismos que intervienen en el desarrollo de agrietamientos poligonales. Para ello se estudian únicamente agrietamientos poligonales formados sobre planos de fractura verticales o subverticales. En particular se establecen relaciones entre la presencia de agrietamientos poligonales y la red de fracturación, la altura de aparición, la orientación e inclinación de la pared, la morfología de las placas y la profundidad de incisión de las grietas perimetrales. Por otra parte, establece relaciones entre procesos geodinámicos internos y procesos de meteorización externos.

Palabras clave: Agrietamientos poligonales; formas graníticas; alteración del granito; Parque Nacional Sierra de Guadarrama.

${ }^{1}$ El presente artículo se enmarca en el proyecto MINECO (CGL2013-40851-P) "Diversidad, bioindicación y biorremediación de protistas en ecosistemas protegidos de paisaje granítico. Hacia estrategias de conservación de especies" (Microepics). 


\title{
Structural control of polygonal cracks in La Pedriza del Manzanares (Madrid)
}

\begin{abstract}
Polygonal cracks represent a common way of modeling granite whose origin and evolution continues under study not even existing a systematization of these diverse structures. Some authors explain their origin by internal geodynamic processes relating to movements of fracture planes in later stages of magmatic consolidation. Other authors attribute their formation and development to external factors related to climate regime. The great variety of polygonal cracks requires the use of a greater number of variables to define their different origins, the possible interrelations between external and internal factors, to explain the evolution of these structures and advance the classification of specific patterns. This work aims to contribute to systematize the mechanisms involved in the development of polygonal cracks. For that only polygonal cracks formed on flat vertical or subvertical fractures are studied. In particular relations are established between the presence of polygonal cracks with: the fracturing network, height of appearance, angle and tilt of the wall, plaque morphology and depth of incision of the perimeter cracks. Moreover it establishes relationships between internal geodynamic processes and external weathering processes.
\end{abstract}

Key words: Polygonal cracking; granite forms; granite weathering; National Park Sierra de Guadarrama.

\section{Contrôle du la structure des fissures polygonales présentes dans La Pedriza de Manzanares (Madrid)}

\section{RÉSUMÉ}

Les fissures polygonales représentent une forme commune de modèles granitiques dont l'origine et l'évolution se trouvent encore en phase d'étude. Certains auteurs de travaux antérieurs expliquent leur origine par des processus internes ayant agi selon des mouvements de plans de fracture dans leur étape finale de consolidation magmatique. D'autres auteurs attribuent leur formation et développement à des facteurs externes en relation avec les conditions climatiques du lieu. La grande variété de fissures polygonales requiert l'utilisation d'une quantité plus importante de variables non seulement pour définir les différentes origines, les interrelations possibles entre les facteurs externes et internes mais aussi pour expliquer l'évolution de ces structures et avancer dans la classification des modèles spécifiques. Cette recherche a pour but de contribuer à systématiser les mécanismes qui interviennent dans le processus de fissures polygonales. À cet effet, l'étude porte uniquement sur les plans de fracture verticaux ou proches de la verticale. Des réactions s'établissent clairement entre l'apparition de fissures polygonales et le réseau de fracturation, la hauteur de leur apparition, l'orientation et l'inclinaison de la paroi ainsi qu'avec la morphologie des plaques et la profondeur d'incision des fissures périmètrales. D'autre part, cette investigation révèle les relations qui existent entre les processus géodynamiques internes et les processus de météorisation externes.

Mots clés: fissures poligonales; formes granitiques; altération de granit; Parc national de la Sierra de Guadarrama. 


\section{INTRODUCCIÓN}

Los agrietamientos poligonales (AP) constituyen un mosaico de placas de geometrías variadas, limitadas por grietas, que recubren parcialmente las superficies de las rocas. Representan un tipo de morfología muy frecuente en los granitoides. Dado que actualmente no existe una sistematización de estas estructuras, agrietamiento poligonal sirve para denominar un amplio abanico de formas que no necesariamente tienen el mismo origen. Hasta el momento las investigaciones atribuyen su origen a factores diversos. Algunos autores relacionan los AP con la etapa final de la consolidación magmática (Vidal Romaní, 1990). Otros los vinculan con el régimen climático (Johnson, 1927; Twidale, 1986; Campbell and Twidale, 1995). Por otro lado, con frecuencia estos factores pueden actuar simultáneamente (Vidal Romaní and Twidale, 2010), o estar relacionados.

Entre los factores destacables que pueden condicionar el origen y la evolución de los AP se encuentran:

- $\quad$ Fenómenos de cizalla producida por movimientos diferenciales entre planos de fractura. La cizalla puede provocar estiramientos y/o acortamientos de la roca creando una fábrica sobre la superficie que favorezca el inicio de las grietas poligonales.

- $\quad$ Factores intrínsecos de la propia roca como son su composición, textura y presencia de fracturas que representan planos de debilidad que favorecen el avance de procesos de meteorización (Leonard, 1929).

El desarrollo de las grietas perimetrales de las placas se produce por meteorización de la roca debida a procesos exógenos. La mayoría de estos procesos tienen relación con el régimen climático por cambios de temperatura, humedad, así como por ciclos de hielo y deshielo. En rocas heterogéneas como el granito, los cristales de cuarzo y feldespato tienen diferentes coeficientes de dilatación térmica y por tanto diferente respuesta ante la meteorización (Hall and André, 2003; Gómez-Heras et al., 2006).

En paredes verticales o subverticales es muy común identificar fracturas más o menos ortogonales a dicha pared que dibujan un enrejado cuadrangular, rectangular, romboidal, o incluso formas más irregulares. Williams and Robinson (1989) explican que el espaciado de las caras de los polígonos depende de la dureza de la roca y de la cantidad de esfuerzo aplicado. Esfuerzos pequeños tienden a producir pocos polígonos grandes, y al contrario, esfuerzos intensos polígonos pequeños y más numerosos.

En esta investigación se estudian AP desarrollados sobre planos de fractura verticales o subverticales en la Pedriza del Manzanares. La finalidad del estudio ha sido buscar un patrón que permita relacionar y tipificar los AP a partir de variables tales como: su altura de aparición desde el suelo, buzamiento de los planos en los que se encuentran, orientación de la red de fracturación preexistente, geometría de las placas de los agrietamientos y profundidad de incisión de las grietas perimetrales. 


\section{ZONA DE ESTUDIO}

La presencia de grietas poligonales es muy frecuente en los granitoides del Sistema Central Español. Buenos ejemplos de AP están presentes en La Pedriza, Zarzalejo, Cenicientos o Valdemanco (García-Rodríguez et al., 2014b y 2014c). Las características petrológicas de estos materiales condicionan los mecanismos y formas de alteración. Así por ejemplo, los monzogranitos de grano medio-grueso de Cenicientos y Zarzalejo presentan mayor disgregación que los constituidos por leucogranitos de grano fino-medio que aparecen en Valdemanco y en la Pedriza (De Pedraza et al., 1989; Pérez-Soba, 1992). Este trabajo se focaliza en la Pedriza, la cual ha sido seleccionada por presentar un tamaño de grano bastante homogéneo y paredes con alturas que superan la decena de metros. Sobre estas paredes es posible definir relaciones entre los AP y su altura de aparición respecto al suelo.

En particular, la zona de estudio ocupa unas $1500 \mathrm{Ha}$ en la Pedriza anterior, área que se extiende desde la localidad de Manzanares el Real hasta la falla del collado de la Dehesilla y del collado Cabrón, con altitudes que oscilan de unos $900 \mathrm{~m}$ en el pueblo de Manzanares el Real, y unos $1700 \mathrm{~m}$ en la cima del Yelmo (Figura 1).

El Sistema Central Español fue la zona axial de la cordillera Ibérica formada durante la orogenia Varisca. Al final de esta orogenia se produjo la intrusión de cuerpos plutónicos en diversas fases, siendo la Pedriza un episodio tardío de granitos altamente fraccionados (Pérez-Soba, 1992; Pérez-Soba y Villaseca, 2010). Después de un largo periodo de arrasamiento, la orogenia Alpina levantó el Sistema Central actual reactivando las fracturas variscas (Capote et al., 1990) y creando el relieve actual. La red de fracturación preferente tiene direcciones E-W,y ENE-WSW y N-S, con superficies de morfologías rectilíneas o curvas (Figura 1).

Figura 1. Vista panorámica de la Pedriza anterior.

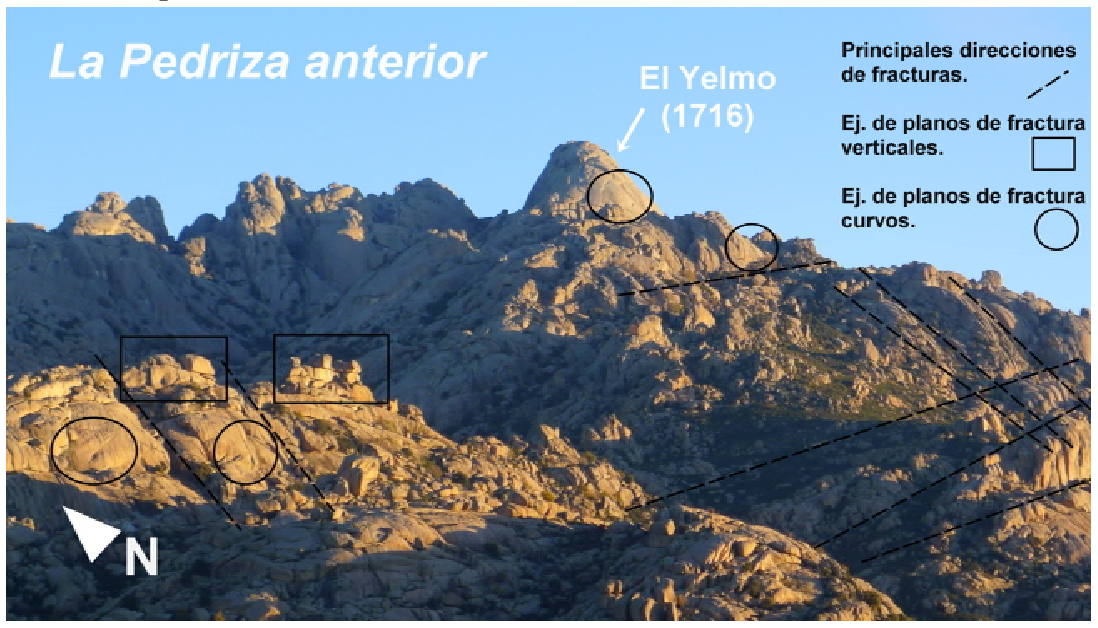

Fuente: Elaboración propia. 
Las fracturas rectilíneas pueden ser verticales, horizontales o inclinadas. Dividen la roca formando un enrejado ortogonal. Los planos curvos de fractura son también muy frecuentes, siendo los responsables de relieves con formas dómicas de altura y verticalidad muy variadas. La dirección predominante de estos planos curvos es E-W con orientación hacia el sur. Por lo general, estas fracturas son más verticales en la base y pierden inclinación en las zonas altas. La gran red de fracturación y la intensa meteorización del macizo rocoso que se inició en condiciones subaéreas, ha permitido que las piedras caballeras sean una forma muy común en la Pedriza. No es difícil reconocer hasta cuatro bloques apilados uno encima de otro.

\section{METODOLOGÍA}

Para alcanzar los objetivos propuestos, una primera fase del trabajo consistió en un minucioso trabajo de campo con la finalidad de identificar e inventariar el mayor número posible de AP desarrollados sobre paredes verticales o subverticales en el área de la Pedriza anterior. Debido a la dificultad para desplazarse por la zona de estudio en muchos casos hubo que recurrir a técnicas de escalada que permitieran acceder a las zonas con AP.

Tabla 1. Inventario de AP sobre planos de fractura.

\begin{tabular}{|c|c|c|c|c|}
\hline Nombre & Coordenadas & $\begin{array}{l}\text { Tipo de plano } \\
\text { (Orientación) }\end{array}$ & $\begin{array}{l}\text { Dirección y } \\
\text { Buzamiento }\end{array}$ & $\begin{array}{c}\text { Altura } \\
\text { desde el } \\
\text { suelo (m) }\end{array}$ \\
\hline Murito & $\begin{array}{l}40^{\circ} 44^{\prime} 59,34^{\prime \prime} \mathrm{N} \\
3^{\circ} 53^{\prime} 17,20^{\prime \prime} \mathrm{W}\end{array}$ & Pared (S) & $\begin{array}{l}\mathrm{N} 80^{\circ} \mathrm{E} \\
70^{\circ} \mathrm{S} / 87^{\circ} \mathrm{S}\end{array}$ & $4-6$ \\
\hline $\begin{array}{l}\text { Pared } \\
\text { Naranja }\end{array}$ & $\begin{array}{l}40^{\circ} 45^{\prime} 08,50^{\prime \prime} \mathrm{N} \\
3^{\circ} 53^{\prime} 10,66^{\prime \prime} \mathrm{W}\end{array}$ & Pared (S) & $\begin{array}{l}\mathrm{N} 60^{\circ} \mathrm{E} \\
85^{\circ} \mathrm{NW}\end{array}$ & $0,5-4,9$ \\
\hline Brezos & $\begin{array}{l}40^{\circ} 45^{\prime} 03,12^{\prime \prime} \mathrm{N} \\
3^{\circ} 53^{\prime} 12,32^{\prime \prime} \mathrm{W}\end{array}$ & $\begin{array}{l}\text { Piedra } \\
\text { Caballera (E) }\end{array}$ & $\begin{array}{l}\mathrm{N} 5^{\circ} \mathrm{E} \\
85^{\circ} \mathrm{E}\end{array}$ & $3-4,25$ \\
\hline Nuez & $\begin{array}{l}40^{\circ} 45^{\prime} 06,54^{\prime \prime} \mathrm{N} \\
3^{\circ} 53^{\prime} 10,31^{\prime \prime} \mathrm{W}\end{array}$ & $\begin{array}{l}\text { Piedra } \\
\text { Caballera (W) }\end{array}$ & $\begin{array}{l}\mathrm{N} 2^{\circ} \mathrm{E} \\
70^{\circ} \mathrm{E}\end{array}$ & $15-20$ \\
\hline $\begin{array}{l}\text { Tres } \\
\text { Puntas }\end{array}$ & $\begin{array}{l}40^{\circ} 44^{\prime} 53,09^{\prime \prime} \mathrm{N} \\
3^{\circ} 53^{\prime} 09,63^{\prime \prime} \mathrm{W}\end{array}$ & Pared $(\mathrm{N})$ & $\begin{array}{l}\mathrm{N} 74^{\circ} \mathrm{E} \\
88^{\circ} \mathrm{N}\end{array}$ & $2-10$ \\
\hline Reloj (S) & $\begin{array}{l}40^{\circ} 44^{\prime} 48,47^{\prime \prime} \mathrm{N} \\
3^{\circ} 52^{\prime} 54,53^{\prime \prime} \mathrm{W}\end{array}$ & Pared (S) & $\begin{array}{l}\mathrm{N} 80^{\circ} \mathrm{E} \\
78^{\circ} \mathrm{S}\end{array}$ & $2-5,5$ \\
\hline Reloj (W) & $\begin{array}{l}40^{\circ} 44^{\prime} 48,47^{\prime \prime} \mathrm{N} \\
3^{\circ} 52^{\prime} 54,53^{\prime \prime} \mathrm{W}\end{array}$ & Pared (W) & $\begin{array}{l}\mathrm{NO}^{\circ} \\
90^{\circ} \mathrm{W}\end{array}$ & $20-25$ \\
\hline Snoopy & $\begin{array}{l}40^{\circ} 44^{\prime} 52,25^{\prime \prime} \mathrm{N} \\
3^{\circ} 52^{\prime} 33,44^{\prime \prime} \mathrm{W}\end{array}$ & Pared (SE) & $\begin{array}{l}\mathrm{N} 48^{\circ} \mathrm{E} \\
71^{\circ} \mathrm{SE}\end{array}$ & $15-20$ \\
\hline Caracol & $\begin{array}{l}40^{\circ} 44^{\prime} 48,96^{\prime \prime} \mathrm{N} \\
3^{\circ} 51^{\prime} 55,63^{\prime \prime} \mathrm{W}\end{array}$ & Pared (S) & $\begin{array}{l}\mathrm{N} 85^{\circ} \mathrm{E} \\
68^{\circ} \mathrm{S}\end{array}$ & $12-15$ \\
\hline Tranco & $\begin{array}{l}40^{\circ} 44^{\prime} 41,98 " \mathrm{~N} \\
3^{\circ} 52^{\prime} 52,09^{\prime \prime} \mathrm{W}\end{array}$ & Pared (W) & $\begin{array}{l}\mathrm{N} 170^{\circ} \mathrm{S} \\
89^{\circ} \mathrm{W}\end{array}$ & $4,5-5$ \\
\hline Martes & $\begin{array}{l}40^{\circ} 45^{\prime} 03,50^{\prime \prime} \mathrm{N} \\
3^{\circ} 51^{\prime} 49,41^{\prime \prime} \mathrm{W}\end{array}$ & Pared (E) & $\begin{array}{l}\mathrm{N} 172^{\circ} \mathrm{E} \\
74^{\circ} \mathrm{W}\end{array}$ & $3-4,5$ \\
\hline $\begin{array}{l}\text { Peñas } \\
\text { Cagás (SE) }\end{array}$ & $\begin{array}{l}40^{\circ} 45^{\prime} 11,90^{\prime \prime} \mathrm{N} \\
3^{\circ} 51^{\prime} 30,17^{\prime \prime} \mathrm{W}\end{array}$ & Pared (SE) & $\begin{array}{l}\mathrm{N} 64^{\circ} \mathrm{E} \\
71^{\circ} \mathrm{SE}\end{array}$ & $20-50$ \\
\hline $\begin{array}{l}\text { Peñas } \\
\text { Cagás (M) }\end{array}$ & $\begin{array}{l}40^{\circ} 45^{\prime} 11,90^{\prime \prime} \mathrm{N} \\
3^{\circ} 51^{\prime} 30,17^{\prime \prime} \mathrm{W}\end{array}$ & Pared $(\mathrm{W})$ & $\begin{array}{l}\mathrm{N} 178^{\circ} \mathrm{E} \\
90^{\circ}\end{array}$ & $30-40$ \\
\hline $\begin{array}{l}\text { Cinco } \\
\text { Cestos }\end{array}$ & $\begin{array}{l}40^{\circ} 45^{\prime} 06,02^{\prime \prime} \mathrm{N} \\
3^{\circ} 52^{\prime} 29,60^{\prime \prime} \mathrm{W}\end{array}$ & $\begin{array}{l}\text { Piedra } \\
\text { Caballera (E) }\end{array}$ & $\begin{array}{l}\mathrm{NO}^{\circ} \\
88^{\circ} \mathrm{E}\end{array}$ & $8-12$ \\
\hline
\end{tabular}

Fuente: Elaboración propia. 
La localización e identificación de zonas con AP se realizó atendiendo a la clasificación de García-Rodríguez et al. (2013) que tiene en cuenta el tipo de superficie sobre la que se encuentran. Es decir, AP localizados en zona superior de fracturas curcas de gran radio, AP situados en la parte inferior de fracturas de gran radio y paredes verticalizadas, o AP desarrollados en planos de fractura verticales o subverticales. En cada zona inventariada con AP se recopiló la siguiente información: nombre que recibe la pared en la que se encuentran, coordenadas de localización y altitud, orientación, dirección y buzamiento de la pared y altura de aparición de los agrietamientos medidos desde el suelo (ver Tabla 1).

Como el objetivo del trabajo era estudiar la presencia de los AP en relación con diferentes variables, se seleccionaron seis paredes tipo (Tabla 2) que permitieran realizar mediciones y establecer un patrón de comportamiento de su aparición.

Tabla 2. Resumen de medidas realizadas en las seis zonas seleccionadas.

\begin{tabular}{|c|c|c|c|c|c|c|}
\hline $\begin{array}{l}\text { Nombre } \\
\text { Risco }\end{array}$ & Altura $(\mathrm{m})$ & Buzamiento & $\begin{array}{l}M(\mathrm{~mm}) \\
\mathrm{m}(\mathrm{mm})\end{array}$ & $(M+m) / 2$ & Forma & $\begin{array}{c}\text { Incisión } \\
\text { (mm) }\end{array}$ \\
\hline \multirow[t]{5}{*}{ Murito } & $6-5,5$ & $70^{\circ} \mathrm{S}$ & $\begin{array}{l}590 \\
342\end{array}$ & 466 & $\begin{array}{l}\text { Cuadrangular } \\
\text { romboidal }\end{array}$ & 10.50 \\
\hline & $5,5-5$ & $85^{\circ} \mathrm{S}$ & $\begin{array}{l}320 \\
260 \\
\end{array}$ & 290 & $\begin{array}{c}\text { Cuadrangular } \\
\text { romboidal }\end{array}$ & 4.20 \\
\hline & $5 \cdot 4,5$ & $87^{\circ} \mathrm{S}$ & $\begin{array}{l}192 \\
160\end{array}$ & 176 & $\begin{array}{c}\text { Cuadrangular } \\
\text { romboidal }\end{array}$ & $4-20$ \\
\hline & 4,5- 4 & $87^{\circ} \mathrm{S}$ & $\begin{array}{c}121 \\
90\end{array}$ & 105 & $\begin{array}{c}\text { Cuadrangular } \\
\text { romboidal }\end{array}$ & - \\
\hline & $4-0$ & $87^{\circ} \mathrm{S}$ & \multicolumn{4}{|c|}{ Sin AP } \\
\hline \multirow[t]{6}{*}{ Snoopy } & 20- 19 & $70^{\circ} \mathrm{SE}$ & $\begin{array}{l}240 \\
168\end{array}$ & 204 & $\begin{array}{c}\text { Cuadrangular } \\
\text { romboidal }\end{array}$ & $40-50$ \\
\hline & 19- 18 & $71^{\circ} \mathrm{SE}$ & $\begin{array}{l}242 \\
174 \\
\end{array}$ & 208 & $\begin{array}{c}\text { Cuadrangular } \\
\text { romboidal }\end{array}$ & $10-20$ \\
\hline & 18- 17 & $70^{\circ} \mathrm{SE}$ & $\begin{array}{l}220 \\
147\end{array}$ & 184 & $\begin{array}{c}\text { Cuadrangular } \\
\text { romboidal }\end{array}$ & $10-20$ \\
\hline & $17-16$ & $72^{\circ} \mathrm{SE}$ & $\begin{array}{l}254 \\
183 \\
\end{array}$ & 184 & $\begin{array}{c}\text { Cuadrangular } \\
\text { romboidal }\end{array}$ & $10-20$ \\
\hline & 16- 15 & $71^{\circ} \mathrm{SE}$ & $\begin{array}{l}214 \\
141\end{array}$ & 141 & $\begin{array}{c}\text { Cuadrangular } \\
\text { romboidal }\end{array}$ & 5. 10 \\
\hline & $15-0$ & $72^{\circ} \mathrm{SE}$ & \multicolumn{4}{|c|}{ Fracturas romboidales pero $\sin \mathrm{AP}$} \\
\hline \multirow[t]{4}{*}{ Martes } & $4,5.4$ & $74^{\circ} \mathrm{W}$ & $\begin{array}{l}210 \\
145\end{array}$ & 177 & $\begin{array}{c}\text { Rectangular } \\
\text { oblicuo }\end{array}$ & $10-25$ \\
\hline & $4-3,5$ & $74^{\circ} \mathrm{W}$ & $\begin{array}{l}157 \\
90\end{array}$ & 124 & $\begin{array}{c}\text { Rectangular } \\
\text { oblicuo }\end{array}$ & $5 \cdot 20$ \\
\hline & $3,5 \cdot 3$ & $74^{\circ} \mathrm{W}$ & $\begin{array}{l}123 \\
85 \\
\end{array}$ & 104 & Irregular & $4-10$ \\
\hline & $3-0$ & $74^{\circ} \mathrm{W}$ & \multicolumn{4}{|c|}{ Sin AP } \\
\hline \multirow[t]{5}{*}{$\begin{array}{l}\text { Pared } \\
\text { Naranja }\end{array}$} & $5-4$ & $85^{\circ} \mathrm{NW}$ & $\begin{array}{l}284 \\
140\end{array}$ & 212 & $\begin{array}{c}\text { Rectangular } \\
\text { vertical }\end{array}$ & $10-20$ \\
\hline & 4-3 & $85^{\circ} \mathrm{NW}$ & $\begin{array}{l}320 \\
155 \\
\end{array}$ & 237 & $\begin{array}{c}\text { Rectangular } \\
\text { vertical }\end{array}$ & $10-20$ \\
\hline & 3.2 & $85^{\circ} \mathrm{NW}$ & $\begin{array}{l}395 \\
177\end{array}$ & 286 & Irregular & $10-20$ \\
\hline & $2-1$ & $85^{\circ} \mathrm{NW}$ & $\begin{array}{l}594 \\
262 \\
\end{array}$ & 428 & $\begin{array}{c}\text { Rectangular } \\
\text { subhorizontal }\end{array}$ & $5-15$ \\
\hline & 1.0 & $85^{\circ} \mathrm{NW}$ & \multicolumn{4}{|c|}{ Sin AP } \\
\hline \multirow[t]{2}{*}{ Tranco } & $5-4,5$ & $89^{\circ} \mathrm{W}$ & $\begin{array}{l}653 \\
27\end{array}$ & 340 & Rectangular & $20-70$ \\
\hline & $4,5-0$ & $89^{\circ} \mathrm{W}$ & \multicolumn{4}{|c|}{ Sin AP } \\
\hline \multirow[t]{3}{*}{ Brezos } & $4,25-3,25$ & $85^{\circ} \mathrm{E}$ & $\begin{array}{l}320 \\
258\end{array}$ & 280 & Irregular & $20-50$ \\
\hline & $3,25-3$ & $85^{\circ} \mathrm{E}$ & $\begin{array}{l}164 \\
128\end{array}$ & 120 & Irregular & 5.30 \\
\hline & $3-0$ & $85^{\circ} \mathrm{E}$ & \multicolumn{4}{|c|}{ Sin AP } \\
\hline
\end{tabular}

Fuente: Elaboración propia. 
Durante la selección de paredes se descartaron las superficies curvas de gran radio, donde los AP presentaban mayor desarrollo y tenían características diferentes de los agrietamientos localizados sobre planos verticales o subverticales. Para garantizar la fiabilidad de las medidas a la hora de establecer relaciones morfométricas entre parámetros en las diferentes zonas con $\mathrm{AP}$, se prestó atención a que ninguna de las zonas inventariadas se hubiera desplazado desde su posición original, por ejemplo que no se hubiera producido volteo en el caso de las piedras caballeras.

Los parámetros seleccionados para caracterizar la frecuencia, orientación y morfometría de los agrietamientos poligonales fueron:

1) Altura de aparición de los agrietamientos respecto a la base de la pared considerada. Con estas medidas se ha intentado buscar relaciones entre la variación del tamaño de los agrietamientos y la incisión de los mismos con la altura y con la distancia al suelo.

2) Buzamiento medio del tramo, que indica la inclinación y sentido del buzamiento de cada tramo de la pared en la que se han localizado agrietamientos poligonales. El objeto de estas medidas ha sido seleccionar paredes con inclinación homogénea, que permitieran establecer relaciones morfométricas entre agrietamientos localizados en paredes con una verticalidad similar. Además del ángulo de buzamiento de la pared, se ha señalado el sentido del buzamiento en relación con la orientación de los agrietamientos. Esta medida ha permitido identificar las paredes con agrietamientos en condiciones de ligero extraplomo y relaciones morfométricas diferentes.

3) Ejes máximos (M) y mínimos (m) medios de las placas de los agrietamientos, medidos perpendicularmente entre sí. En este trabajo se presentan los valores medios de las medidas de los ejes máximos y mínimos de los intervalos de altura seleccionados en cada pared. Los valores medios se han obtenido siempre entre 5 y 9 medidas por tramo, según la abundancia de formas presentes en cada tramo y pared. La altura de los intervalos en los que se han medido agrietamientos ha oscilado entre $0,5 \mathrm{~m}$ y $1 \mathrm{~m}$ dependiendo de la altura de la pared y la superficie total de agrietamientos presentes sobre la pared.

4) Para facilitar el análisis de datos se ha utilizado un índice de forma que relaciona los ejes mayor y menor medidos perpendicularmente, según la relación $(\mathrm{M}+\mathrm{m}) / 2$. Este índice permite, con un único valor por tramo, representar cómo varía el tamaño del agrietamiento con la altura.

5) Forma geométrica que presenta el agrietamiento en cada tramo. Puede ser cuadrangular, romboidal, rectangular o irregular. La descripción de la forma del agrietamiento se ha realizado para establecer la relación de las orientaciones de las caras de los agrietamientos, con el sistema de fracturas que afecta a la pared.

6) Profundidad de la incisión de las grietas perimetrales de las placas en cada tramo altitudinal. Esta medida permite conocer cómo varía la profundidad de la grieta en relación con la altura respecto al suelo. 
Para comprobar si la morfología de los agrietamientos se dispone en función del sistema de fracturas que intersectan con la pared en la que se encuentran, se han medido los ángulos que forman las lineaciones de las fracturas con las caras de los agrietamientos. La medida del ángulo se ha realizado a partir de fotografías ortogonales a la pared. Un ángulo de $0^{\circ}$ indica que la lineación coincide con la cara del agrietamiento, mientras que un ángulo de $90^{\circ}$ representa la diferencia angular máxima entre lineación de las fracturas y agrietamiento. Estas medidas se han realizado en cinco de los emplazamientos seleccionados, no habiendo sido posible realizarlas en el "Cancho de los Brezos" debido a la irregularidad de las caras de los agrietamientos.

Para conocer si existe alguna relación entre la altura de aparición de los agrietamientos medida desde el suelo y el tamaño de los agrietamientos expresados como un índice de forma, se han representado en un mismo gráfico los datos medios de los índices de forma de cada intervalo y de cada pared. El caso de obtener alineaciones verticales $u$ horizontales indicaría que son factores independientes, y el caso de darse alineaciones inclinadas querría decir que sí habría relación entre ambos factores.

Con el propósito de cuantificar cuánto es de fuerte la relación entre el índice de forma y la altura, se ha empleado el coeficiente de correlación de Pearson, que varía entre -1 y 1 . Un valor de 0 indicaría que no hay relación entre los fenómenos estudiados. Un valor de 1 indicaría una relación directa (en que ambos factores crecen o decrecen a la par) y perfecta. Finalmente un valor de -1 indicaría una relación perfecta e inversa, cuando un factor aumenta el otro disminuye. Es decir, en caso de haber varios factores relevantes en la determinación del tamaño de los agrietamientos, el coeficiente de Pearson nos habla de la importancia tiene el factor altura con relación a otros posibles factores.

Por último, con objeto de conocer cuál es el peso de la relación entre altura e índice de forma, es decir, el porcentaje de varianza del factor a explicar (el tamaño de los agrietamientos) en base al factor explicativo (la altura), se ha calculado el coeficiente de determinación. Un coeficientes de determinación con un valor $\mathrm{r}^{2}=0$ indicaría que la variabilidad del fenómeno a explicar (el índice de forma) no tiene ninguna relación con la variación de la altura en que se dan dichos agrietamientos. Para que se entienda mejor, por ejemplo un valor $\mathrm{r}^{2}=0,80$, supondría que para esa pared, aproximadamente el $80 \%$ de la variabilidad del índice de forma estaría asociada a la variación de la altura (no se trata de un caso real en nuestro estudio).

\section{RESULTADOS Y DISCUSIÓN}

Los agrietamientos poligonales presentes en la Pedriza de Manzanares pueden incluirse en alguno de los dos tipos siguientes:

Agrietamientos formados sobre diques de rocas intrusivas que rellenan fracturas del granito.

Agrietamientos desarrollados sobre planos de fractura que afectan a la propia roca granítica. 
De los agrietamientos del segundo tipo, en La Pedriza del Manzanares se han identificado tanto sobre planos verticales - subverticales $\left(90^{\circ}-70^{\circ}\right)$, como en grandes superficies curvas con un buzamiento inferior a $70^{\circ}$, correspondientes a planos de fractura de gran radio (García-Rodríguez et al., 2014a y 2014c).

Según se apuntaba en el apartado de metodología, durante el trabajo de campo se seleccionaron paredes con AP desarrollados sobre planos de fractura verticales o subverticales. Se descartaron AP formados en superficies curvas de gran radio, que por norma general, están mucho más desarrollados que los identificados sobre planos verticales, y por tanto no son comparables.

Los planos que forman las paredes con agrietamientos poligonales presentan una red ortogonal de fracturas con dirección W-E o WSW-ENE y N-S. Estas fracturas forman paredes con alturas que varían desde unos pocos metros, hasta algo más de la centena de metros. Los muros que tienen mayor altura suelen están orientados hacia el sur y con buzamiento en el mismo sentido sur. La abundancia de fracturas de dirección W-E es precisamente la responsable del relieve escalonado que presenta la Pedriza anterior en la vertiente orientada hacia el pueblo de Manzanares el Real.

Algunos ejemplos de paredes con nombre propio localizadas en la zona de trabajo y con alturas comprendidas entre unos 80 y 150 m, son: las placas del Halcón, placas de Musgogénesis, el Reloj, el muro de Snoopy, las placas del Tranco, Peñas Cagás y Cinco Cestos. El número de paredes o muros verticales con alturas comprendidas entre unos 3 o $4 \mathrm{~m}$, y hasta cerca de la $40 \mathrm{~m}$ es innumerable. Algunos ejemplos son: el Murito, la Nuez, la pared Naranja, los Brezos, Tres Puntas y el Tranco.

Del análisis de la tabla 1 se deduce que los agrietamientos más abundantes y mejor desarrollados presentan orientación sur - sureste (46,6\% de los casos), seguidos de los de orientación oeste $(26,6 \%)$ y este $(20 \%)$. Menos frecuentes son los agrietamientos con orientación norte $(6,6 \%)$. Estos resultados, aunque todavía algo preliminares, parecen señalar el importante papel de la insolación en la formación de AP, al ser las orientaciones sur que tienen mayor radiación solar, las que presentan mayor número de estas formas.

En todos los casos el desarrollo de agrietamientos no llega a los niveles inferiores del afloramiento. Pensamos que esto puede deberse al tiempo de exposición en condiciones aéreas, mayor en las zonas de más altura, donde la acción de los agentes que favorecen el deterioro (insolación-humedad), ha podido actuar durante un periodo de tiempo mayor (García-Rodríguez et al., 2015).

La morfología de las placas de agrietamientos suelen presentar formas cuadrangulares (en un $10 \%$ de los casos), rectangulares (23\%), romboidales $(7 \%)$ o irregulares $(60 \%)$. La profundidad de las hendiduras que separan cada placa es mayor en las zonas superiores y de menor pendiente (Tabla 2). Por otra parte, la incisión de los agrietamientos está condicionada por la red de fracturación secundaria ortogonal a la pared, y el máximo desarrollo de la incisión tiene relación con dichas fracturas preexistentes (Figura 2). 
Figura 2. Formas de los agietamientos poligonales ${ }^{2}$.
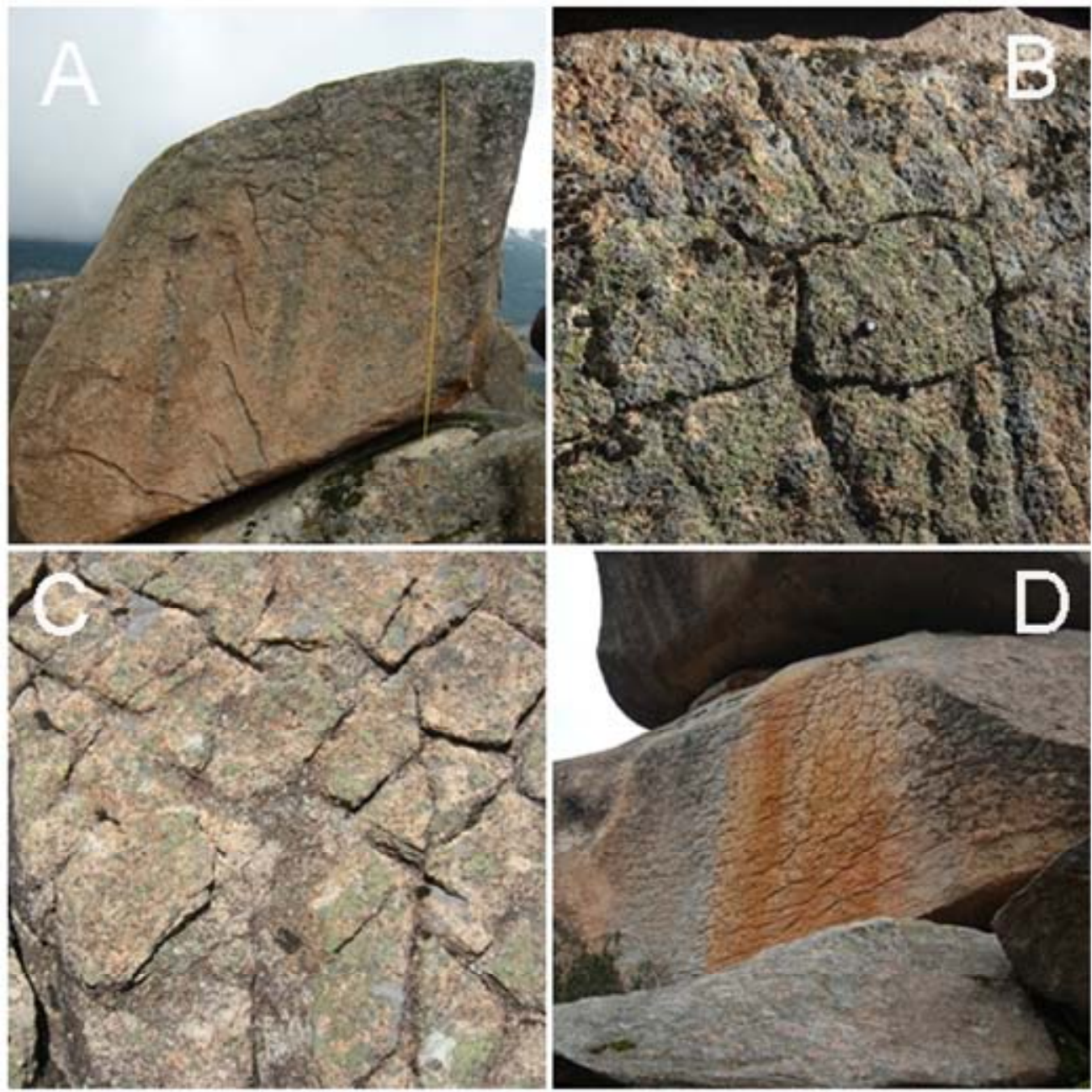

Fuente: Elaboración propia.

La identificación de agrietamientos poligonales sobre determinadas piedras caballeras (bloque de los Brezos, la Nuez o cinco Cestos) en las que los planos de fractura originales son perfectamente reconocibles, ha permitido constatar una relación directa entre planos de fractura y formación de agrietamientos.

2 A) AP irregulares en una piedra caballera en los Brezos, B) AP cuadrangulares y rectangulares en el Murito, C) AP cuadrangulares y romboidales en el muro de Snoopy, D) AP cuadrangulares en la pared Naranja. 
En la mayoría de los afloramientos analizados se ha encontrado una relación directa entre la orientación (lineación) del sistema de fracturas y su espaciado, con la forma del agrietamiento. Incluso en algún caso, se han identificado agrietamientos con caras curvas, adaptándose a fracturas curvas secundarias perpendiculares a la pared vertical (Risco del Martes). Esta tesis sobre la relación de la morfología de los agrietamientos y el sistema de fracturas se ha puesto a prueba en este estudio. En la tabla 3A se muestra, para cada pared, y para todas de forma promediada, el ángulo menor entre las lineaciones de fracturas y la cara de la placa que forma el agrietamiento. Un ángulo con un valor muy pequeño indicaría que el agrietamiento está muy alineado con el sistema de fracturas. La tabla 3A no incluye el bloque de los Brezos, que por la irregularidad que presentan las caras de los agrietamientos ha resultado imposible realizar mediciones del ángulo que forman con las lineaciones presentes en la pared.

Tabla 3. Relaciones angulares y de índices de forma de AP.

\begin{tabular}{|c|c|c|c|c|}
\hline \multirow{2}{*}{$\begin{array}{l}\text { Nombre } \\
\text { de la } \\
\text { pared }\end{array}$} & \multirow[t]{2}{*}{ A) } & \multirow{2}{*}{$\begin{array}{l}\text { Angulo entre líneas de } \\
\text { fractura y caras de } \\
\text { agrietamientos }(N=44)\end{array}$} & \multicolumn{2}{|c|}{$\begin{array}{l}\text { B) Indices de forma en relación a la altura } \\
\text { de la pared }\end{array}$} \\
\hline & & & $\begin{array}{c}\text { Correlación de } \\
\text { Pearson }\end{array}$ & $\begin{array}{l}\text { Coeficiente de } \\
\text { determinación }\end{array}$ \\
\hline Murito & & 3,90 & 0,55 & 0,31 \\
\hline Snoopy & & 7,80 & 0,40 & 0,16 \\
\hline Martes & & 5,63 & 0,57 & 0,32 \\
\hline Naranja & & 5,63 & $-0,75$ & 0,56 \\
\hline Tranco & & 4,88 & - & - \\
\hline Brezos & & - & 0,76 & 0,58 \\
\hline
\end{tabular}

Fuente: Elaboración propia.

Según la tabla $3 \mathrm{~A}$ un valor de ángulo alto indicaría que el agrietamiento está dispuesto de forma discrepante al sistema de fracturas y supondría que la morfología del agrietamiento, no va pareja a dicho sistema de fracturas. Como los valores obtenidos oscilan entre un mínimo de $3,90^{\circ}$ a un máximo de $7,80^{\circ}$, con una media de $4,47^{\circ}$, las mediciones indican un alineamiento agrietamiento - sistema de fracturas alto. En el mismo sentido, la figura 3 representa las mediciones del ángulo que forman los agrietamientos con el sistema de fracturas pared a pared y mediante datos agrupados por intervalos. El número de medidas en las que el ángulo entre agrietamiento y fracturas es muy similar (inferior al $5^{\circ}$ ) es muy alto para cada uno de los afloramientos estudiados, lo que corroboran la hipótesis de formación del alineamiento entre agrietamientos y sistemas de fracturación. El verde representa valores que corroboran máximamente la hipótesis del alineamiento entre agrietamientos y sistemas de fracturación. Los colores que se van acercando al rojo, representan los datos que cada vez corroboran menos la tesis mencionada. 
Figura 3. Angulo entre lados de AP y fracturas ${ }^{3}$.

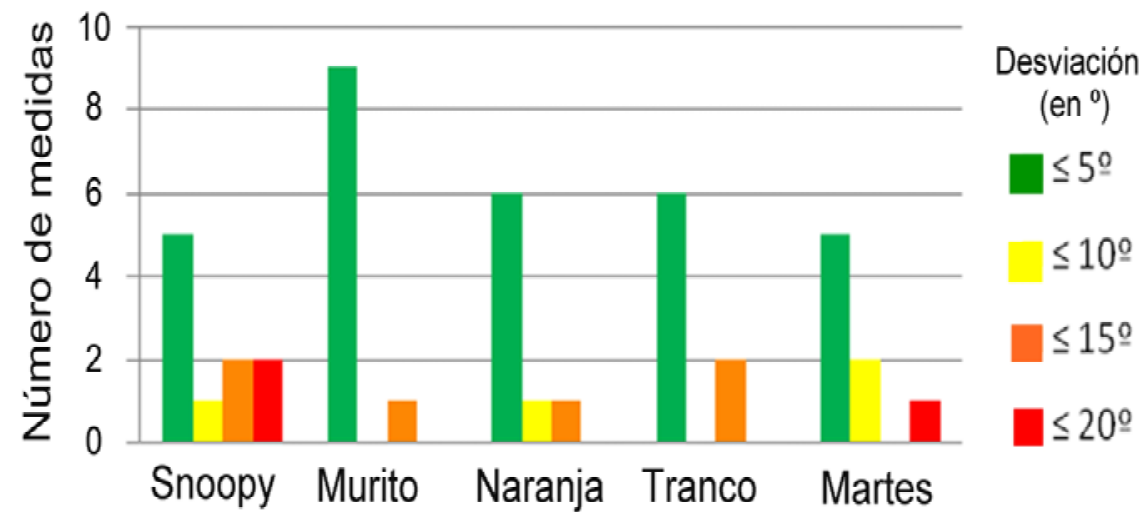

Fuente: Elaboración propia.

Al analizar la relación entre el factor de forma y la altura de aparición de los agrietamientos, en el Murito, Muro de Snoopy, Risco del Martes y en el Bloque de los Brezos, el índice de forma aumenta con la altura. La pared Naranja presenta una relación inversa respecto a las anteriores. En el bloque de cancho de camino del Tranco no es posible establecer relaciones por disponer únicamente de un intervalo de medidas en una altura. Esta relación puede cuantificarse y explicarse a partir de los valores de la correlación de Pearson (Tabla 3B), que muestran la relación entre el tamaño del agrietamiento y la altura, con valores que van desde $r=0,40$ hasta $r=$ 0,76. La correlación es positiva en el Muro de Snoopy, Risco del Martes, y Brezos. En el caso de la pared Naranja, la relación es fuerte e inversa, con un valor de $r=-$ 0,75 donde las dimensiones de las placas se incrementan desde las zonas superiores a las inferiores del afloramiento.

A partir de las observaciones de campo y de los resultados presentados, la anomalía que presenta la pared Naranja respecto a las otras paredes, podría explicarse si se tiene en cuenta que tiene un buzamiento hacia el NW, en sentido contrario a la orientación de la pared, creando un ligero extraplomo. Además, la presencia de un bloque de piedra sobre la pared y la presencia de óxidos, podrían actuar como elementos distorsionadores de la insolación y de la escorrentía del agua de lluvia que circula sobre su superficie.

\footnotetext{
${ }^{3}$ Se representa el número de medidas identificadas en cada emplazamiento con agrietamientos poligonales, con indicación de la desviación en grados entre la dirección de las fracturas y la dirección de los lados de los agrietamientos.
} 
Por otra parte, al calcular el coeficiente de determinación (Tabla 3B) se han obteniendo 3 grupos con diferente comportamiento, que aportan cierta información sobre los resultados de la correlación de Pearson. En el primer grupo se incluye únicamente el "Muro del Snoopy" en el que la variación de la altura explica sólo el $16 \%$ la variación en índice de forma. Eso supone que el 84\% restante se debe a otros factores. El segundo grupo está formado por el "Murito" y el "Martes", en los que con un $31 \%$ y $32 \%$ respectivamente de la variación del índice de forma, se podría predecir correctamente a partir de la variación de la altura. En este caso la variación explicada es mayor que en el Muro de Snoopy, por lo que el peso de este factor es más determinante en estas 2 paredes. El tercer grupo estaría formado por la Pared Naranja y los Brezos, en las que la varianza en índice de forma de los agrietamientos asociada a la varianza de altura es del $56 \%$ y $58 \%$ respectivamente. Estos resultados apoyan la idea de que en estas 2 paredes la altura es un factor fundamental en el índice de forma de los agrietamientos.

Analizando los resultados del coeficiente de determinación de todas las paredes en conjunto se interpreta que la gran diferencia entre los valores obtenidos en el primer y segundo grupo, puede explicarse por la gran diferencia existente de la altura de aparición de los agrietamientos. Los agrietamientos del muro de Snoopy se localizan entre 10 y $15 \mathrm{~m}$ por encima de los del grupo dos, indicando una exposición a la intemperie durante mucho más tiempo que en los otros. Por otra parte, y apoyando esta misma idea, en la Pared Naranja y en los Brezos, la varianza del índice de forma de los agrietamientos asociada a la varianza de altura es la mayor, coincidiendo que se tratándose de los agrietamientos localizados a menor altura con respecto al suelo.

Al promediar los coeficientes de determinación de las distintas paredes se obtiene un valor de $r^{2}=0,39$, es decir, que para estas paredes, aproximadamente el $40 \%$ de la variabilidad del índice de forma está asociada a la variación de la altura en que se encuentran dichos agrietamientos. Este dato indica la importante relevancia de la altura en la comprensión de las características geomorfológicas consideradas, y que cuanto mayor es la altura de aparición de los agrietamientos, más disminuye el coeficiente de determinación. Indica también, la necesidad de ampliar en estudios posteriores la comprensión del fenómeno para, considerando finalmente un conjunto de variables relevantes, tratar de alcanzar niveles de predicción de la variable a explicar lo más próximos al 100\% que sea posible.

\section{CONCLUSIONES}

Existe una amplia variedad de agrietamientos poligonales, que además de los factores internos y externos que los originan, tiene relación con el tipo de afloramiento sobre el que se localizan, bien sobre bloques, o en paredes de tamaño y verticalidad variada. Las diferentes variables que intervienen en su desarrollo no permiten definir un único patrón que explique el origen de todos los agrietamientos poligonales. En este trabajo se han estudiado AP sobre planos de fractura verticales y subverticales, y establecido una línea de comportamiento en la que se relaciona la red de fracturación del macizo rocoso, la altura de aparición de los AP, el tamaño de las 
placas y la profundidad de incisión de las grietas perimetrales de las placas. En general, los resultados muestran que los agrietamientos desarrollados sobre este tipo de paredes, tienen mayor tamaño de placa y profundidad de incisión de las grietas en las zonas de más altura. Por otra parte, los resultados obtenidos establecen relaciones entre procesos geodinámicos internos y procesos de meteorización externos a favor de planos de debilidad preexistentes.

\section{BIBLIOGRAFÍA}

CAMPBELL, E.M., TWIDALE, C.R. (1995). Lithologic and climatic convergence in granite morphology. Cadernos Laboratorio Xelóxico de Laxe 20:381-403.

CAPOTE, R., DE VICENTE, G., GONZÁLEZ CASADO, J.M. (1990). Evolución de las deformaciones alpinas en el Sistema Central Español. Geogaceta 7: 20-22.

DE PEDRAZA J., SANZ MA., MARTÍN A. (1989). Formas graníticas de la Pedriza. Agencia de Medio Ambiente. Comunidad de Madrid. 205 pp.

GARCÍA-RODRÍGUEZ, M., CENTENO J.D, GÓMEZ-HERAS, M., FORT GONZÁLEZ, R., ALVAREZ DE BUERGO, M., (2013). Thermal and structural controls on polygonal cracking in granite of La Pedriza de Manzanares (Spain). Abstracts Volume 8th International Conference (AIG) on Geomorphology. Geomorphology and Sustainability. Paris, 27-31 August 2013, p. 303.

GARCÍA-RODRIGUEZ, M., GARCÍA RODRÍGUEZ, M., SALCEDO MIRANDA, J.L. (2014a). "El Hueso" de la Pedriza: origen, estructura y rasgos geomorfológicos.Tecnologi@y Desarrollo, UAX. Vol. XII. 20 pp.

GARCÍA-RODRÍGUEZ, M., GÓMEZ-HERAS, M., FORT GONZÁLEZ, R., ALVAREZ DE BUERGO, M., CENTENO, J.D., (2014b). Influencia de los endurecimientos superficiales en el micro-relieve de las superficies graníticas de La Pedriza de Manzanares. Parque Nacional de Guadarrama (España). Tecnologi@y Desarrollo, UAX. Vol. XII. 23 pp.

GARCÍA-RODRÍGUEZ, M., GÓMEZ-HERAS, M., ALVAREZ DE BUERGO, M., FORT GONZÁLEZ, R., CENTENO, J.D., (2014c). Caracterización de agrietamientos poligonales sobre granito en La Pedriza de Manzanares y en Cenicientos, Madrid (Sistema Central). Revista M+A, 15(1):22-36.

GARCÍA-RODRÍGUEZ, M., GÓMEZ-HERAS, M., FORT GONZÁLEZ, R., ALVAREZ DE BUERGO, M. (2015). Control térmico de la meteorización de superficies endurecidas en rocas graníticas (La Pedriza de Manzanares, España). Boletín de la Sociedad Geológica Mexicana. En prensa.

GOMEZ-HERAS, M., SMITH, B.J., FORT, R. (2006) Surface temperature differences between minerals in crystalline rocks: Implications for granular disaggregation of granites through thermal fatigue. Geomorphology 78 (3-4): 236249.

HALL, K, ANDRÉ, M.F. (2003). Rock thermal data at the grain scale: applicability to gr.anular disintegration in cold environments. Earth Surf Process Landf 28: 823-836. 
JONHSON, R.J. (1927). Polygonal weathering in igneous and sedimentary rocks. American Journal of Science 13: 440-444.

LEONARD, R.J. (1929). Polygonal cracking in granite. American Journal of Science 18: 487-492.

PÉREZ-SOBA, C. 1992. Petrología y geoquímica del macizo granitico de La Pedriza, Sistema Central Español. Doctoral Thesis. Madrid, Universidad Complutense de Madrid, $222 \mathrm{pp}$.

PÉREZ-SOBA, C., VILLASECA, C. (2010). Petrogenesis of highly fractionated Itype peraluminous granites: La Pedriza pluton (Spanish Central System). Geologica Acta 8: 131-149.

TWIDALE, C.R. (1986). Granite landform evolution: factors and implications. Geologische Rundschau 75: 769-779.

VIDAL ROMANÍ, J.R. (1990). Formas menores en rocas graníticas: un registro de su historia deformativa. Cadernos do Laboratorio Xeolóxico de Laxe 15: 317-328.

VIDAL ROMANÍ, J.R., TWIDALE, CR. (2010). Structural or climatic control in granite landforms? The development of sheet structure, foliation, boudinage, and related features. Cadernos do Laboratorio Xeolóxico de Laxe 35:189-208.

WILLIAMS RBG, ROBINSON DA. (1989). Origin and distribution of polygonal cracking of rocks surfaces. Geografiska Annaler. Series A, Physical Geography 71: 145-159. 